\title{
Superresolution via enhanced evanescent tunneling
}

\author{
Alessandro Salandrino* and Demetrios N. Christodoulides \\ Center for Research and Education in Optics and Lasers (CREOL)/College of Optics and Photonics, \\ University of Central Florida, Orlando, Florida 32816, USA \\ *Corresponding author: a.salandrino@gmail.com
}

Received November 30, 2010; revised January 11, 2011; accepted January 12, 2011; posted January 14, 2011 (Doc. ID 138998); published February 7, 2011

\begin{abstract}
We here propose the concept of enhanced evanescent tunneling (EET). Our analysis indicates that by means of a suitable control field, the transmission of evanescent waves across a forbidden gap can be enhanced by several orders of magnitude-well beyond the ordinary frustrated total internal reflection case. We show how such a phenomenon can be used to probe both the amplitude and phase of the evanescent portion of the angular spectrum, thereby allowing target superresolution. In principle EET can be manifested in other areas of physics where wave tunneling is involved. (c) 2011 Optical Society of America

OCIS codes: $240.7040,260.2110$.
\end{abstract}

The resolution limitations of conventional far-field optics are well known and they are fundamental by nature. The physics of free space electromagnetic propagation itself prevents the possibility of resolving features beyond the so-called Abbe-Rayleigh limit [1]. The information associated with subwavelength details of an illuminated object is in fact carried by evanescent waves, which decay exponentially with distance and are eventually lost. In the past decades several new ideas have been put forward in order to alleviate or entirely circumvent the shortcomings of conventional imaging. Despite the success of near-field optics [2], the pursuit of a far-field subdiffraction microscope never stopped. Interesting and effective contributions came from the most diverse perspectives [3-ㅜㄹ].

In this Letter we introduce the concept of enhanced evanescent tunneling (EET) and show how it can be used to probe both amplitude and phase of the evanescent portion of the angular spectrum of a field distribution. This process effectively exploits the peculiar features of the evanescent waves themselves. This is accomplished through an auxiliary beam that is appropriately matched to the signal-thus boosting the power tunneled over a forbidden gap. As opposed to other arrangements, this effect can be employed to extract the evanescent information even when direct contact with the target is impossible, e.g., when it is submerged a few wavelengths below the accessible surface.

The exponential decay of the evanescent part of the target's angular spectrum is ultimately responsible for limiting the resolution of an ideal far-field imaging system. Such shortcomings may be overcome if and only if a scheme is devised that enables one to measure these cut-off components. In a lossless medium, the spatial evolution of evanescent waves along the direction of decay is such that the net power flow is zero. More specifically the vanishing of the real part of the Poynting vector is due to the $90^{\circ}$ relative phase between the electric and magnetic field components-orthogonal to the decay direction. Nevertheless, instances exist in which evanescent waves can support a net power flow, as for example in the case of frustrated total internal reflection (FTIR), which is illustrated schematically in Fig. 1(a). In this case two dielectric half-spaces having equal relative permittivities $\varepsilon_{1}$ are separated by a gap of lower permittivity $\varepsilon_{2}$ and of length $d$. Assuming that a TE polarized plane wave is incident upon the first interface under total internal reflection, then the electric field distribution in the intermediate layer is given by the superposition of a "direct" evanescent wave of amplitude $A_{D} \in \Re$ decaying from the first interface and a "reflected" evanescent wave of amplitude $A_{R}$ decaying away from the second interface. Note that neither of the two evanescent fields can separately contribute to the power transfer across the gap. Yet, the cooperative action of the two evanescent components does lead to a net power flow in the $z$ direction, which in terms of the Poynting vector reads $\mathbf{S} \cdot \hat{\mathbf{z}}=\alpha /\left(k_{0} \eta_{0}\right) \exp (-\alpha d) A_{D} \operatorname{Im}\left[A_{R}\right]$, where $\eta_{0}$ is the vacuum characteristic impedance, $k_{0}=\omega / c$, and $\alpha$ is the evanescent wave decay constant.

The presence of the interference factor $A_{D} \operatorname{Im}\left[A_{R}\right]$ in this expression lends itself to a simple interpretation of the physics behind this power flow. Depending on the relative phase of the coefficients $A_{D}$ and $A_{R}$, the magnetic field of the reflected wave can provide a component that is in phase with the electric field of the direct wave. Hence, the electric field of the direct wave $A_{D}$ can "ride" across the gap by using the in-phase component of the reflected magnetic field, proportional to $\operatorname{Im}\left[A_{R}\right]$.

The concept of EET emerges here as a way of imitating the power transfer mechanism of FTIR in a fully controllable fashion, without its intrinsic limitations. The main idea behind EET is to apply an auxiliary control field so as to induce a net power flow from an evanescent signal. Let us then consider a similar geometry in the presence of an additional control field, as shown in Fig. 1(b). Two applied mutually coherent fields given by
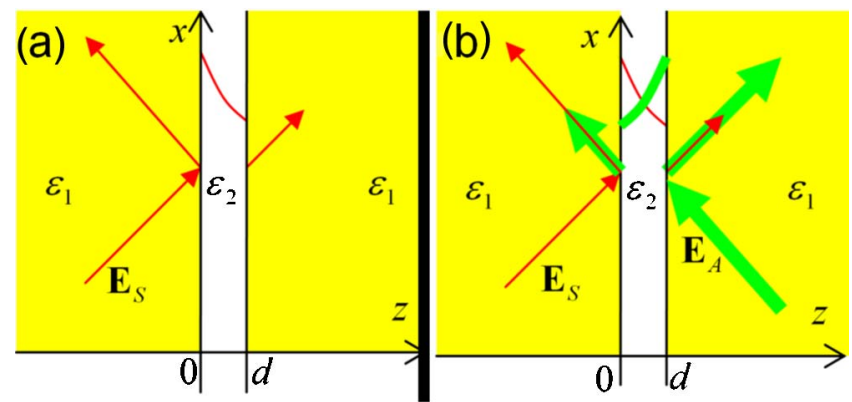

Fig. 1. (Color online) (a) Evanescent tunneling through a gap by FTIR, (b) EET. 
$\mathbf{E}_{S}=\hat{\mathbf{y}} A_{S} e^{i k_{z} z} e^{i k_{x} x}$ and $\mathbf{E}_{A}=\hat{\mathbf{y}} A_{A} e^{i \Delta \varphi_{A S}} e^{-i k_{z}(z-d)} e^{i k_{x} x}$ are incident at the same angle at $z=0$ and $z=d$. The coefficients $A_{S}$ and $A_{A} \in \Re$ represent the amplitude of the signal and of the auxiliary fields, respectively, with $\Delta \varphi_{A S}$ being their relative phase. The field in the gap is now a superposition of the evanescent waves produced by the total internal reflection of both the signal and the auxiliary field.

It can be shown that the combination of the two evanescent fields will sustain a net power flow across the structure, given by

$$
\begin{aligned}
P_{z}= & \frac{2 k_{z}^{2} \alpha}{k_{0} \eta_{0}} \frac{k_{z} \alpha\left(A_{S}^{2}-A_{A}^{2}\right)}{\left[2 k_{z} \alpha \cosh (\alpha d)\right]^{2}+\left[\left(k_{z}^{2}-\alpha^{2}\right) \sinh (\alpha d)\right]^{2}} \\
& +\frac{2 k_{z}^{2} \alpha}{k_{0} \eta_{0}} \frac{\left(k_{z}^{2}+\alpha^{2}\right) \sinh (\alpha d) A_{S} A_{A} \sin \left(\Delta \varphi_{A S}\right)}{\left[2 k_{z} \alpha \cosh (\alpha d)\right]^{2}+\left[\left(k_{z}^{2}-\alpha^{2}\right) \sinh (\alpha d)\right]^{2}} .
\end{aligned}
$$

Three different components are identified in Eq. (1). The quadratic term in $A_{S}$ describes the power transfer due to the FTIR of the signal alone. The analogous quadratic term in $A_{A}$, bearing a negative sign, is associated with an FTIR energy flow in the opposite direction. More interesting is the mixed term proportional to $A_{A} A_{S}$, which will be referred to as "Poynting interference." This term accounts for the cooperative power transfer effects enabled by the electric field of one wave and the in-phase magnetic field component of the other and is maximum for $\Delta \varphi_{A S}=\pi / 2$. The direction of this Poynting interference is solely determined by the relative phase $\Delta \varphi_{A S}$ of the two evanescent fields involved, regardless of their amplitude.

A careful consideration of this property indeed reveals counterintuitive consequences, in particular in the regime for which the Poynting interference dominates over the FTIR terms. Figure 2 illustrates the power flow across a $3-\mu$ m-long air gap separating two silicon $(n=3.5)$ halfspaces in a configuration similar to that of Fig. 1(b), as a function of the auxiliary field power. Two electromagnetic waves of wavelength $1.5 \mu \mathrm{m}$ are incident on the two interfaces at an angle of $20^{\circ}$ with respect to the nor$\mathrm{mal}$, so as to be in total internal reflection mode. To demonstrate this effect, let us assume that the power density of the signal is kept at $1 \mathrm{~W} / \mathrm{m}^{2}$ while the auxiliary field power density is varied over a broad range. The relative phase between the signal and the auxiliary field is optimally set at $\Delta \varphi_{A S}=\pi / 2$. For the sake of comparison, in the absence of the auxiliary field the power density flux in the $z$ direction that could tunnel across the structure by FTIR would be $1.28 \times 10^{-7} \mathrm{~W} / \mathrm{m}^{2}$.

Intuitively one would expect that if the auxiliary field is larger than the signal, power would flow in the negative $z$ direction. On the other hand, Fig. 2 shows that there is a whole region where the auxiliary field is literally orders of magnitude larger than the signal and yet power flows across the gap in the positive $z$ direction. Such an anomaly is a consequence of Poynting interference, shown in red in Fig. 2. This effect indeed overcomes the auxiliary field FTIR contribution (shown in green in Fig. 2) in the low-intensity regime and enhances the tunneling of the signal.

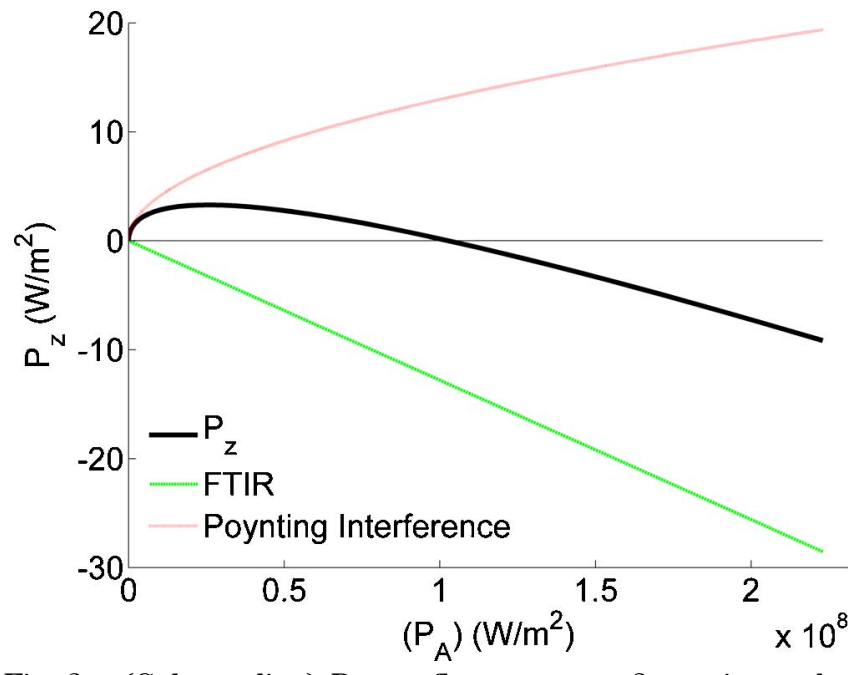

Fig. 2. (Color online) Power flow across a $3 \mu \mathrm{m}$ air gap between two silicon half-spaces.

It can be directly shown that maximum EET occurs when the amplitude of the auxiliary field is given by $A_{A}=A_{S} \sinh (\alpha d) \sin \left(\Delta \varphi_{A S}\right)\left(k_{z}^{2}+\alpha^{2}\right) /\left(2 k_{z} \alpha\right)$, provided that $\Delta \varphi_{A S}=\pi / 2$. Under these conditions the power flow across the gap attains a maximum value of $P_{\mathrm{MAX}}=$ $\left(k_{z} / 2 k_{0} \eta_{0}\right) A_{S}^{2}$. It is important to notice that a net increase in the total power transfer across the gap occurs if and only if the signal and the auxiliary field share the same transverse wave vector. If this condition is not met, the Poynting interference contribution averages to zero over the illuminated area.

The question naturally arises as to whether the EET effect could be used to boost the evanescent spectrum of an illuminated object in order to resolve subdiffraction features. A schematic of a possible EET imaging setup is shown in Fig. 3. This simple arrangement consists of a dielectric hemispherical dome of refractive index $n$ brought into proximity to a backilluminated sample. Clearly, for the EET effect to take place it is important that both the illumination field and the auxiliary wave are mutually coherent. The subdiffraction features of the object would in this case be associated with evanescent waves in the region between the sample and the dielectric hemisphere. In principle, if this gap were absent, i.e., if the sample could be placed in direct contact with the dome, a portion of the evanescent angular spectrum would tunnel into the dielectric region and be converted

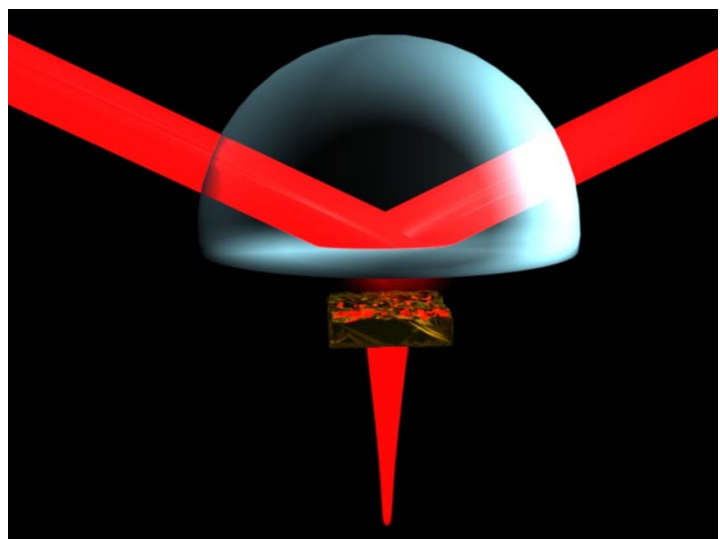

Fig. 3. (Color online) EET in an imaging setup. 
into propagating waves. Unfortunately, in many experimental situations, depending on the structure of the sample, this might not be a viable option.

In such instances the EET could effectively allow measurement of both amplitude and phase of a portion of the evanescent spectrum in order to improve the resolution of the optical instrument. In the configuration shown in Fig. 3, the auxiliary evanescent field is produced by the total internal reflection of a broad beam at the planar interface of the dielectric dome. In order to probe the evanescent spectrum scattered by the sample, different spatial harmonics have to be selectively enhanced. This can be achieved by scanning the auxiliary beam in azimuth and elevation, so as to phase match the appropriate evanescent component. Upon tunneling, the enhanced spatial harmonic will overlap with the reflected auxiliary beam. The resulting amplitude of the total Poynting vector (normal to the dome) can be expressed as

$$
\begin{aligned}
I= & \frac{n A_{A}^{2}}{2 \eta_{0}}+\frac{2 e^{-2 \alpha d} n \alpha^{2} A_{S}^{2}}{\left(k_{z}^{2}+\alpha^{2}\right) \eta_{0}} \\
& +\frac{2 e^{-\alpha d} n \alpha\left[k_{z} \sin \left(\Delta \varphi_{A S}\right)-\alpha \cos \left(\Delta \varphi_{A S}\right)\right]}{\left(k_{z}^{2}+\alpha^{2}\right) \eta_{0}} A_{A} A_{S} .
\end{aligned}
$$

Three components can be identified in Eq. (2). The first term, quadratic in $A_{A}$, is the irradiance contribution due to the auxiliary wave reflection, and is clearly the dominant term. The second term in the expression [Eq. (2)] is quadratic in the spatial harmonic amplitude $A_{S}$ and accounts for the "unaided" tunneling of the signal scattered by the sample. This contribution is by far the smallest and decreases with the sample distance $d$ as $\exp (-2 \alpha d)$. The effect of EET is described by the third term in Eq. (2), which is proportional to the product $A_{A} A_{S}$. This signal term carries all the information concerning the subwavelength features of the target, and can be effectively extracted from the background reflection of the auxiliary beam using homodyning or heterodyning schemes (provided the sample illumination is modulated). Such a contribution can be enhanced by merely increasing the amplitude $A_{A}$ of the auxiliary field and by properly tuning the relative phase $\Delta \varphi_{A S}$ between the auxiliary field and the corresponding phase-matched spatial harmonic of the signal. We would also like to point out that by virtue of heterodyne detection, the scattering of the auxiliary field from the sample can be isolated from the useful signal, and was therefore omitted in Eq. (2). It is important to notice that the EET achieves a maximum when $\Delta \varphi_{A S}=\pi / 2+\arctan \left(k_{z} / \alpha\right)$. By scanning the phase of the auxiliary field until such maximum condition is reached, it is possible to determine the phase of the enhanced spatial harmonic of the sample. The corresponding amplitude follows by simple inversion of the following relation:

$$
I_{\mathrm{EET}}=\frac{4 e^{-\alpha d} \alpha^{2} k_{z} n}{\eta\left(\alpha^{2}+k_{z}^{2}\right)^{3 / 2}} A_{A} A_{S}
$$

By scanning the incidence angle of the auxiliary field, it is in principle possible with this method to extend the collected angular spectrum up to maximum transverse wave vectors of amplitude $k_{0} n$. This additional information, once boosted by the auxiliary beam, could therefore allow one to resolve details $n$ times smaller than in a conventional optical instrument. From a practical perspective, this theoretical resolution will ultimately be limited by the available power in the auxiliary beam. In other words, the amplitude of the auxiliary field necessary for optimum EET increases with the angle of incidence to be probed. Note that the strong auxiliary wave will decay at the interrogation site and thus is expected to be harmless to the target.

The resolution that can be achieved by EET is certainly limited by the refractive index of the dielectric, as in the case of a solid immersion lens [10], but with an important difference. Simply increasing the permittivity of the sample's environment cannot lead to an increased resolution, unless the features to be resolved are in direct contact with the high-refractive-index medium. An air gap of a fraction of a wavelength would be enough to lose almost completely the evanescent components, nullifying the effects of a solid immersion lens. The advantage of EET is to bridge the lower-index gaps between the target and the high-permittivity dome. This arrangement can be employed in the visible using high-index GaP domes.

Similar results are obtained for the TM case, with a Poynting vector given by

$$
\begin{aligned}
I= & \frac{\eta_{0} A_{A}^{2}}{2 n}+\frac{2 \eta_{0} e^{-2 \alpha d} n^{3} \alpha^{2} A_{S}^{2}}{\left(k_{z}^{2}+\alpha^{2} n^{4}\right)} \\
& +\frac{2 \eta_{0} e^{-\alpha d} n \alpha\left[k_{z} \sin \left(\Delta \varphi_{A S}\right)-\alpha n^{2} \cos \left(\Delta \varphi_{A S}\right)\right]}{\left(k_{z}^{2}+\alpha^{2} n^{4}\right)} A_{A} A_{S},
\end{aligned}
$$

where $A_{S}$ and $A_{A}$ refer to the amplitude of the transverse magnetic fields involved. In this case, the Poynting interference is maximized when $\Delta \varphi_{A S}=\pi-\arctan \left[k_{z} /\left(\alpha n^{2}\right)\right]$.

In conclusion, the concept of EET has been introduced, and possible applications for subdiffraction imaging have been proposed. We emphasize that this enhancement or boosting of the evanescent signal is made possible by exploiting the peculiar nature of the evanescent waves themselves. What we describe here is entirely passive and makes no use of any amplification (via stimulated emission, etc.).

\section{References}

1. E. Abbe, Arch. Mikrosk. Anat. 9, 413 (1873).

2. E. A. Ash and G. Nicholls, Nature 237, 510 (1972).

3. S. W. Hell and J. Wichmann, Opt. Lett. 19, 780 (1994).

4. X. Zhuang, Nat. Photon. 3, 365 (2009).

5. A. Pertsinidis, Y. Zhang, and S. Chu, Nature 466, 647 (2010).

6. A. Salandrino and N. Engheta, Phys. Rev. B 74, 075103 (2006)

7. Z. Jacob, L. V. Alekseyev, and E. Narimanov, Opt. Express 14, 8247 (2006).

8. Z. Liu, H. Lee, Y. Xiong, C. Sun, and X. Zhang, Science 315, 1686 (2007).

9. S. Gazit, A. Szameit, Y. C. Eldar, and M. Segev, Opt. Express 17, 23920 (2009).

10. Q. Wu, G. D. Feke, R. D. Grober, and L. P. Ghislain, Appl. Phys. Lett. 75, 4064 (1999). 